\title{
An overview of genotype $x$ environment interaction and yield stability analysis in applied plant breeding: great emphasis given to coffee (Coffea arabica $\mathrm{L}$.)
}

\author{
Wakuma Merga Sakata*iD
}

Received 29 October 2021, Revised 11 November 2021, Accepted 20 December 2021, Published online 31 December 2021

\begin{abstract}
A B S T R A C T
The inconsistence of genotypes across location during plant breeding is the major challenges to the breeder. That is the differential response of genotypes to different environment. Meanwhile stability is the ability of a genotype to withstand stressful conditions and yet be able to produce yield. Thus, stability is an absolute and relative measure. Arabica coffee has location specific adaptation nature and that leads to highly significant instability in its breeding program. In the study of coffee bean yield stability cultivars tested at multilocations within the domain of coffee growing ecologies of Ethiopia, showed a significant genotype $\mathrm{x}$ environment interaction. The review of previous research also indicated inconsistent effects of genotype $\mathrm{x}$ environment interaction on cup quality. Yield-stability analysis is very important in measuring cultivar stability and suitability for growing crops across seasons and agro-ecological region to identify stable genotype. The yield stability have been challenge to the plant breeders and biometricians, it complicates the selection of superior genotypes. It is important to minimize the usefulness of the genotype across environments for selecting. Since approach of plant breeding is to develop genotypes that are, optimum for the condition under which they will be grown breeders have to manage yield instability throughout formalized procedures of plant breeding. During stability measurement if the variance is found to be significant, various methods of measuring the stability of genotypes can be used to identify the stable genotype(s). Most of stability analysis parameters are briefly discussed in this review.
\end{abstract}

Keywords: Arabica coffee, GGE bi-plot, Static stability coefficient and Yield stability index

Ethiopian Institute of Agricultural Research, Teppi Agricultural Research Center, P.O.Box: 34 Teppi, Ethiopia

*Corresponding author's email: wakumerga@gmail.com (W.M. Sakata)

Cite this article as: Sakata, W.M. 2021. An overview of genotype x environment interaction and yield stability analysis in applied plant breeding; great emphasis given to coffee (Coffea arabica L.). Int. J. Agril. Res. Innov. Tech. 11(2): 117-123. https://doi.org/10.3329/ijarit.v11i2.57264

\section{Introduction}

Genotype by environment interaction refers to the differential responses of genotypes across environments (Bavandpori et al., 2015). It is important factor to be studied in plant breeding. The repeatable genotype by environment interactions resulted change the ranking of genotypes across environments, and are meaningful for the specific breeding strategy (Sabaghnia et al., 2008). It is common for economically importance quantitative traits. A biometrical genetics definition of GEI refers to a genotype-specific sensitivity to environmental exposure of an organism (Fisher et al., 1932). They were not convinced that GEI was an important influence on traits and treated it as a nuisance because they found that he could remove GEI through a simple transformation of the scale of the environment.
Genotype $\mathrm{x}$ environment interaction and yieldstability analysis has continued to be important in measuring varietal stability and suitability for cultivation across seasons and ecological zones. The analyses of genotype $\mathrm{x}$ environment has focused on the identification of stable genotypes for cultivation. According to Cooper et al. (2001), magnitude of genotype by environmental interaction is higher where there is wide variation between environments. It can be micro or macro, non-organic or organic, and internal or external.

Plant breeding identifies causes of GEI towards predictability, separate predictable from unpredictable, GEI Separate genotype and environment components of the GEI Structured models. Several statistical models have been used to understand interactions of different crops for identifying suitable genotypes (Teressa et al., 2021). These differences and the ensuing debate highlighted certain assumptions and limitations 
of the biometrical genetics approach to GEI. Initial GEI research may not have addressed the nature of genetic and environmental influences to appropriately reflect processes related to human outcomes. The initial studies of GEI using breeding experiments generally assumed genetic and environmental influences to function independently of one another.

GEI and yield stability have been challenge to the breeders and biometricians for a long time because it complicates the selection of superior genotypes by reducing the genetic progress. GEI is important to minimize the usefulness of the genotype means across locations or environments for selecting and advancing superior genotypes to the next stage of selection (De Leon et al., 2016). Plant breeders have managed these interactions throughout the history of crop domestication, crop improvement, and dispersal, and within recent history through the formalized procedures of plant breeding.

In Ethiopia, the relationship between selection environments and target production environment had been a fundamental problem because many of the selected activities performed by the conventional approach are in on research stations, which are good production environments (Melkamu et al., 2015). Many statistical approaches consider all of the phenotypic variation (i.e., means across environments), which may be misleading. GEI is not merely a problem, it is also an opportunity" (Simmonds, 1991). The varietal stability could be challenged not only due to the change in the test environment but also due to change in growing season per environment. Some environmental variations are predictable and others may be unpredictable. In Ethiopia, coffee thrives under a wide range of environmental conditions. It grows best at altitudes between 1000 and 2000 meters and it grows wild in many parts of the country (Dereje and Eshetu, 2012). Conscious selection of superior agro types adapted to a range of complex agro ecologic situations did not receive priority in the coffee improvement programme of the country until after the outbreak of CBD (Coffee berry disease, Colletotrichum coffeanum) in 1971. It was then felt necessary to design the cultivar evaluation program to cover a wide range of varieties and locations. The objective of this paper is to review Genotype by Environmental interaction and stability analysis in plant breeding by giving more emphasis to Arabica coffee.

\section{Genotype $x$ environment interaction in coffee (Coffea arabica L.)}

Significant genotype-environmental interactions for yield of coffee have been reported by several researchers (Walyaro, 1983; Afework, 2017; Lemi et al., 2018) They stressed on the need for testing coffee over a wide range of ecological conditions before making decisions either for specific or extensive use of cultivars. In his study, Walyaro (1983) revealed that selecting high yielding genotypes with linear responses to environments was possible. The Standing Committee on Coffee Research of the Institute of Agricultural Research and a consultant scientist in effect therefore, advocated this. Their judgments were because none of the coffee berry disease resistant cultivars so far planted across Ethiopia performed uniformly across the many very different environmental zones in which coffee is widely grown. When differences between environments are large, a character measured under such conditions is regarded as more than one character. A high positive correlation between characters in different environments will reflect high heritability of the major character complex involved (Mesfin and Beyyata, 2003).

The variation in genotypic response from one environment to another is an intrinsic part of a genotypic behavior and without its estimation, assessment of a genotype remains incomplete. Some researchers have been studied this phenomenon in Ethiopia and they tried to specify and estimate the stability and adaptability of many Arabica coffee characters and their response to changing environments however the information regarding the GEI is not consistent due to different genotypes evaluated at different location.

In the study of bean yield stability of coffee cultivars tested at multi locations in Ethiopia within the domain of the main coffee growing ecologies of the country, showed a significant effect of location $\mathrm{x}$ genotype interaction indicating differential response of genotypes across the many different locations (Mesfin and Bayeta, 1997). They stated that none of the cultivars tested across the many different locations showed linear response with changes in environmental conditions showing their specificity only for defined ecological zones. Based on their study, they stressed on the need for testing coffee cultivars over a wide range of ecological conditions before making decision either for specific or extensive use of cultivars. Similar studies in other countries by different workers have also indicated the presence of strong significant genotype $\mathrm{x}$ environment interaction (Agwanda et al., 1997) in Coffea arabica L. However, these authors at the same time indicated the presence of stable cultivars in the population of their Coffea arabica materials. They concluded that selecting high yielding genotypes with a linear response to changes in environment is possible. Similarly, Montagnon et al. (2000) also reported the presence of strong genotype by environment interaction in Coffea canephora but with some stable cultivars that stabilize coffee bean yield in Coffea canephora materials. 
Other researchers (Agwanda et al., 2003) reported significant genotype $\mathrm{x}$ environment interaction effects on coffee bean and liquor quality. On the other hand, Walyaro (1983) reported relatively lower genotype $\mathrm{x}$ environment interaction effects on quality characters. Currently Lemi et al. (2018) reported there is significant genotype by environment interaction for bean yield and organoleptic quality in coffee arabica studied in Ethiopia at different location. Sivetz (1963) indicated that if other factors are kept equal, better quality coffee could be found at higher altitudes. Wellman (1961) reported that lowland coffee was somewhat bland, with considerable body, while coffee from high altitude areas was more acidic, with better aroma and flavour. Review of previous research results indicated inconsistent effects of genotype $x$ environment interaction on cup quality. Therefore, quality evaluations based on multi-site trials could also be used to identify environments that best reveal differences in genetic potentials amongst varieties and hence useful as selection and/or test sites (Agwanda et al., 2003). This could improve the efficiency with which selection for superior quality could be attained.

\section{The concept of stability in plant breeding}

The goal of any plant breeding is to produce genotypes that are, in some sense, optimum for the condition under which they will be grown. One approach is to develop genotypes that are widely adapted over wide range of environmental condition. These stable genotypes provide a stock from which superior genotypes may be selected. Although it is important to detect genotypes by environment interaction by conducting yield trials over a series of environments, this alone gives no measure of the stability of individual genotypes. Hence, stability measurements are important since they give an indication of the adaptability of genotypes to general or specific condition.

Stability is the ability of a genotype to produce or perform under stressful conditions and yet be able to respond (Lin et al., 1986). Tollenaar and Lee (2002) defined stability as a measure of the ability of a genotype to maintain relative performance across wide environments. Genotypes that show little interaction with environments are called stable. Stability is either a static or a dynamic where in static, performance of the genotype remains unchanged regardless of the environmental conditions and in dynamic, performance of a genotype changes in a predictable manner across a wide range of environmental conditions (Tollenaar and Lee, 2002). Thus, static stability is an absolute measure, while dynamic stability is a relative measure.

The term "stability of genotypes" is central to all types of analyses of GEI especially with reference to plant breeding. Researchers use the terms adaptation, phenotypic stability and yield stability in different ways (Becker and Léon, 1988). Stability in common usage connotes consistency in performance that would mean minimum variation among environments for a particular genotype (Chahal and Gosal, 2002).

The stability with which a plant breeder is concerned implies stability in those aspects of phenotype, which are important economically, such as grain yield and quality. Such stability may depend upon holding some aspects of morphology and physiology in a steady state but allowing others to vary. In this way, the desirable varieties will show low GEI interaction for agriculturally important characters, especially grain yield, but not necessarily for other characteristics. Two basic concepts of phenotypic stability are distinguished: the biological concept, and the dynamic concept.

The biological concept of stability refers to the constant performance of a genotype over a wide range of environments. As to Becker and Léon (1988) in static stability a genotype, possess unchanged performance regardless of variation of the environments, thus implying that its variance among environments is zero. This type is seldom a desired feature of crop cultivars, since no response to improved growing conditions would be expected. On the other hand, dynamic stability, also termed as agronomical concept of stability, implies that a stable genotype should always give high yield expected at the level of productivity of the respective environments. With quantitative traits, the majority of genotypes often react similarly to favorable or unfavorable environmental conditions. Becker and Léon (1988) stated that all stability procedures based on quantifying GEI interaction effects belong to the dynamic stability concept. This includes the procedures for partitioning the GEI of ecovalence and Shukla's (1972) stability of variance, procedures using the regression approach such as proposed by Finlay and Wilkinson (1963), Eberhart and Russell (1966) and Perkins and Jinks (1968), as well as non-parametric stability statistics.

\section{Statistical methods to measure GEI and yield stability}

A combined analysis of variance procedure is the most common method used to identify the existence of GEI from replicated multiplication trials. If the GEI variance is found to be significant, one or more of the various methods for measuring the stability of genotypes can be used to identify the stable genotype(s). A wide range of methods is available for the analysis of GEI. Among the several stability parameters proposed by different authors, the most popular are briefly discussed in the following 
subheadings. Those are, ecovalence (Wi) of Wricke model, Shukla's variance stability model $\left(\delta^{2} \mathrm{i}\right)$, cultiver superiority measure (Pi) of Lin and Binns modal, additive main effects and multiplicative interaction (AMMI) model, genotype main effect and GEI (GGE) bi-plot, AMMI stability value, static stability coefficient and yield stability index.

\section{Additive main effects and multiplicative interaction method (AMMI)}

According to Zobel et al. (1988), considering the three traditional models, ANOVA fails to detect a significant interaction component, PCA fails to identify and separate the significant genotype and environment main effects, and linear regression models accounts for only a small portion of the interaction sum of squares. However, AMMI analysis reveals highly significant interaction components. The AMMI method is used for three main purposes. The first is model diagnoses, it is more appropriate in the initial statistical analysis of yield trials, because it provides an analytical tool of diagnosing other models as sub cases when these are better for particular data sets (Gauch, 1988). Secondly, AMMI clarifies the GEI and it summarizes patterns and relationships of genotypes and environments. The third use is to improve the accuracy of yield estimates. Gains have been obtained in the accuracy of yield estimates that are equivalent to increasing the number of replicates by a factor of two to five (Crossa, 1990). Such gains may be used to reduce testing cost by reducing the number of replications, to include more treatments in the experiments or to improve efficiency in selecting the best genotypes. The AMMI model combines the analysis of variance for the genotype and environment main effects with principal components analysis of the GEI. It has proven useful for understanding complex GEI. The results can be graphed in a useful bi-plot that shows both main and interaction effects for both genotypes and environments. AMMI combines analysis of variance (ANOVA) into a single model with additive and multiplicative parameters. The principal components analysis of AMMI partitions GEI into several orthogonal axes, the IPCA. Gauch and Zobel (1996) showed that IPCA 1 against IPCA 2 is generally informative.

Since AMMI has the bi-plot feature, genotypes and environments are plotted on the same diagram, facilitating inference about specific interactions of individual genotypes and environments by using the sign and magnitude of PCA 1 values. Any genotype with a PCA 1 value close to zero shows general adaptation to the test environment. A large genotypic PCA 1 scores reflects more specific adaptation to environments with PCA 1 scores of the same sign. Integrating bi-plot display and genotypic stability statistics enable genotypes to be grouped based on similarity of performance across diverse environments. Plant breeders can easily select from a bi-plot those entries that are high yielding and stable, and those entries that yield well at specific sites (Yau, 1995. Different authors used this model for their study (Afework, 2017; Lemi et al., 2018)

\section{Genotype main effect and GEI (GGE) bi- plot}

Yan (2002) proposed a methodology known as genotype and genotype by environment (GGE) bi-plot for graphical display of GEI pattern of MET data with many advantages. GGE bi-plot analysis considers both genotype and GEI effects and graphically displays GEI in a two-way table (Yan and Hunt, 2001). It is an effective method based on principal component analysis (PCA) to fully explore MET data. It allows visual examination of the relationships among the test environments, genotypes and the GEI. A bi-plot is a scatter plot that graphically exhibits a point for each genotype and each environment (Gabriel, 1971). Bi-plot have been used to identify "which-wins where" patterns. The genotype, which is located at the corner of one polygon, is the best performer in that environment included in that sector. Environment that is located far away from the origin discriminate the genotypes more than those near the origin do. It is effective tool for Mega-environment analysis, whereby specific genotypes can be recommended to specific mega-environments, Genotype evaluation, estimation of yield and stability of genotypes were done by using the average environment (Yan and Hunt, 2001) and to testenvironmental evaluation.

\section{AMMI stability value (ASV)}

AMMI Stability Value (ASV) is the distance from the coordinate point to the origin in a twodimensional plot of IPCA 1 scores against IPCA 2 scores in the AMMI model (Purchase, 1997). Because the IPCA 1 score contributes more to the GXE interaction sum of squares, a weighted value is needed. This weighted value was calculated for each genotype and each environment according to the relative contribution of IPCA 1 to IPCA 2 to the interaction sum of squares. Genotypes with lower ASV values are considered more stable than genotypes with higher ASV. The ASV, which uses two IPC scores to produce a balanced measurement between them, can be useful in situations where the two first IPCs accounted for considerable amount of GE interactions (Anley et al., 2013). AMMI stability value, which is stability value based on the AMMI model's IPCA 1 and IPCA 2 values for each genotype and each environment, was calculated as suggested by Purchase et al. (2000). 


\section{Cultivar superiority measure (Pi) of Lin} and Binns model

The superiority measure (Pi) of cultivars is estimated by the squares of differences between genotype's and maximum genotype mean, summed and divided by twice the number of locations (Lin and Binns, 1988). The method has great advantage of an immediate recommendation of more stable and adapted genotypes, due to the uniqueness of the parameter, the evaluation of genotype performance according to the environmental variation and the fact that the genotypes identified as the most stable and adapted are generally the most productive. The most stable genotype is the one with least deviation from the maximum yield of each environment, i.e., with the lowest $\mathrm{Pi}$ value. It measures mean performance and stability simultaneously. Different authors such as, (Afework, 2017 and Lemi et al., 2018) on coffee used this stability parameter to identify high yielding and stable genotypes across different locations.

\section{Static stability coefficient (SSC)}

The static stability coefficient is defined as the variance around the genotype's phenotypic mean across all environments. This provides a measure of the consistency of the genotype, without accounting for performance. It is based on environmental variances i.e. the variance of yields of each genotype over test environments (Becker and Leon, 1988). A low value of this coefficient indicates a better fit of a genotype to the static stability concept. Lemi et al. (2018) used this stability parameter to evaluate stability of Limmu coffee genotypes.

\section{Ecovalence (Wi) of Wricks model}

Wricke (1964) proposed that the concept of ecovalence using the contribution of each genotype to the GEI sum of squares as stability measure and defined this concept or statistics as ecovalence (Wi). According to this model, genotypes with low value of Wi have smaller deviations from the mean across environments and are thus more stable. The lower the value of $\mathrm{Wi}$, the smaller will be the fluctuations from the predictable response in different environments so that the genotype with the least $\mathrm{Wi}$ is considered to be ideal from the point of view of yield stability. According to Becker and Leon (1988), genotype with zero ecovalence is regarded as stable. Lemi (2016) used this stability parameter for evaluation of $C$. arabica genotypes.

\section{Shukla's variance stability model ( $\left.\boldsymbol{\delta}^{\mathbf{2}} \mathrm{i}\right)$}

Shukla (1972) developed the concept of stability variance of genotype $i$ as its variance across environments after the main effects of environmental means have been removed. Since, the genotype main effect is constant; the stability variance is thus based on the residual (GEij+ eij) matrix in a two-way classification. The estimated value of Shukla's stability variance $\left(\delta^{2} i\right)$ can be negative since the stability variance is the difference between two sum of squares and the value of this negative estimates of variance $(\delta)$ is taken as equal to zero (Shukla, 1972). Lin and Binns (1986) reported that Shukla's stability variance is a relative measure that depends on the genotype in the test and thus the results must be restricted to only those genotypes in the test and should not be generalized. Therefore, a genotype is considered as stable genotype when its contribution to the total GEI sum of squares is small as compared to the contribution of other genotypes in a given test.

\section{Yield stability index (YSI)}

Farshadfar et al. (2011) developed this parameter. Stability should not be the only parameter for selection, because the most stable genotypes would not necessarily give the best yield performance. Hence, there is a need for approaches that incorporate both mean yield and stability in a single index, that is why various authors introduced different selection criteria for simultaneous selection for yield and stability. In this regard, as ASV takes into account both IPCA 1 and IPCA 2 that justify most of the variation in the GEI. The rank of ASV and yield mean in such a way that the lowest ASV takes the rank one, while the highest yield mean takes the rank one and then the ranks are summed in a single simultaneous selection index of yield and yield stability named as yield stability index (YSI). The least YSI is considered as the most stable with high bean yield.

\section{Summary and Conclusion}

The ultimate goal of plant breeders in a crop improvement program is development of varieties with high yield and stability. In Arabica coffee variety development program there is high GEI. Therefore, it is very important to reduce the genotype means across different environments. It is a continues task of plant breeders because of the environmental fluctuation. The main purpose of multi-environment trials is to observe stability of genotypes across the environments, the identification of superior genotypes and of the location, which best represents the target environment for production. An approach of breeder is to develop genotypes that are widely adapted over wide range of environmental condition. These stable genotypes provide a stock from which superior genotypes selected. Genotypes that show little interaction with environments are stable. It is either a static or a 
dynamic where in static, performance of the genotype remains unchanged regardless of the environmental conditions and dynamic, performance of a genotype changes in a predictable manner across a wide range of environmental. Plant breeders have to manage yield instability throughout formalized procedures of plant breeding. During stability measurement if the GEI variance is found to be significant, various methods of measuring the stability of genotypes can be used to identify the stable genotype. A combined analysis of variance procedure is the most common method used to identify the existence of GEI.

\section{References}

Afework, L. 2017. Genotype x environment interaction and stability analysis of some promising Ilu Ababora Coffee (Coffea arabica L.) genotypes for yield and yield related traits in Southwestern Ethiopia. M.Sc. Thesis, Department of Horticulture and Plant Science, Jimma University, Ethiopia. pp. 68-90.

Agwanda, C.D., Baradat, P.C. and Charrier, A. 1997. Genotype by environment interaction and its implication. In: XvlleColloqueScintifique International due café, Nairobi, Kenya. pp. 424-429.

Agwanda, C.O., Baradat, P., Eskes, A., Cilas, C. and Charrier, A. 2003. Selection for bean and liquor qualities within related hybrids of Arabica coffee in multilocal field trials. Euphytica. 131(1): 1-14. https://doi.org/10.1023/A:1023071815109

Anley, W., Zeleke, H. and Dessalegn, Y. 2013. Genotype $\mathrm{X}$ environment interaction of maize (Zea mays L.) across North Western Ethiopia. J. Plant Breed. Crop Sci. 5(9): 171181. https://doi.org/10.5897/JPBCS2013.0406

Bavandpori, F., Ahmadi, J. and Hossaini, S.M. 2015. Yield stability analysis of bread wheat lines using AMMI model. Agril. Comm. 3(1): 8-15.

Becker, H. and Leon, J. 1988. Stability analysis in plant breeding. Plant Breed. 101(1): 1-23. https://doi.org/10.1111/j.1439-0523.1988.tboo261.x

Chahal, G.S. and Gosal, S.S. 2002. Principles and procedures of plant breeding: biotechnological and conventional approaches. Alpha Science International Limited. p. 126.

Cooper, M., Woodruff, D.R., Phillips, I.G., Basford, K.E. and Gilmour, A.R., 2001. Genotype-by-management interactions for grain yield and grain protein concentration of wheat. Field Crops Res. 69(1): 47-67. https://doi.org/10.1016/So378-4290(oo)o0131-3

Crossa, J. 1990. Statistical Analyses of Multilocation Trials. Adv. Agron. 44: 55-85. https://doi.org/10.1016/So065-2113(08)60818-4
De Leon, N., Jannink, J.L., Edwards, J.W. and Kaeppler, S.M., 2016. Introduction to a special issue on genotype by environment interaction. Crop Sci. 56(5): 2081-2089. https://doi.org/10.2135/cropsci2016.07.0002in

Dereje, G. and Eshetu, A. 2012. Crop and agroecological zone of Ethiopia. Academia. p. 32.

Eberhart, S.A. and Russell, W.A. 1966. Stability parameters for comparing varieties. Crop Sci. 6: 36-40.

https://doi.org/10.2135/cropsci1966.0011183Xo 00600010011x

Farshadfar, E., Zali, H. and Mohammadi, R. 2011. Evaluation of phenotypic stability in chickpea genotypes using GGE-Bi-plot. Annal. Biol. Res. 2(6): 282-292.

Finlay, K.W. and Wilkinson, G.N. 1963. The analysis of adaptation in a plant-breeding programme. Australian J. Agril. Res. 14(6): 742-754. https://doi.org/10.1071/AR9630742

Fisher, R.A., Immer, F.R. and Tedin, O. 1932. The genetical interpretation of statistics of the third degree in the study of quantitative inheritance. Genetics. 17(2): 107-124. https://doi.org/10.1093/genetics/17.2.107

Gabriel, K.R. 1971. The bi-plot graphic display of matrices with application to principal component analysis. Biometrika. 58(3): $453-467$.

https://doi.org/10.1093/biomet/58.3.453

Gauch Jr, H.G. 1988. Model selection and validation for yield trials with interaction. Biometrics. 44: 705-715. https://doi.org/10.2307/2531585

Gauch Jr, H.G. and Zobel, R.W. 1996. Optimal replication in selection experiments. Crop Sci. 36(4): 838-843.

https://doi.org/10.2135/cropsci1996.0011183Xo 03600040002x

Lemi, B. 2016. Genotype by environment interaction and stability analysis of advanced limmu coffee (Coffea arabica) genotypes in Southwestern Ethiopia. MS Thesis, Department of Horticulture and Plant Science, Jimma University, Ethiopia. p. 68.

Lemi, B., Alamerew, S., Ayano, A. and Daba, G. 2018. Genotype environment interaction and yield stability of Arabica coffee (Coffea arabica L.) genotypes. African J. Agril. Res. 13(4): 210-219.

https://doi.org/10.5897/AJAR2017.12788

Lin, C.S. and Binns, M.R. 1988. A superiority measure of cultivar performance for cultivar x location data. Canadian J. Plant Sci. 68(1): 193-198. https://doi.org/10.4141/cjps88-018

Lin, C.S., Binns, M.R. and Lefkovitch, L.P. 1986. Stability analysis: where do we stand? Crop Sci. 26: 894-900.

https://doi.org/10.2135/cropsci1986.0011183Xo 02600050012x 
Melkamu, T., Sentayehu, A. and Eticha, F. 2015. GGE biplot analysis of genotype by environment interaction and grain yield stability of bread wheat genotypes in South East Ethiopia. World J. Agril. Sci. 11(4):183190.

Mesfin, A. and Bayetta, B. 1997. Genotypeenvironment interaction and its implication on selection for improved quality in arabica coffee (Coffea arabica L.) genotypes. ASIC, 17e Colloque, Nairobi, Kenya.

Mesfin, A. and Beyyata, B. 2003. Institute of Agricultural Research Coffee improvement Division, Jimma Research Centre, Addis Ababa Ethiopia.

Montagnon, C., Cilas, C., Leroy, T., Yapo, A. and Charmetant, P. 2000. Genotype-location interactions for Coffea canephora yield in the Ivory Coast. Agronomie. 20(1): 101-109. https://doi.org/10.1051/agro:2000110

Perkins, J.M. and Jinks, J.L. 1968. Environmental and genotype-environmental components of variability III. Multiple lines and crosses. Heredity. 23: 339-356. https://doi.org/10.1038/hdy.1968.48

Purchase, J., Hatting, H. and Van Deventer, C. 2000. Genotype $\mathrm{x}$ environment interaction of winter wheat ( $T$. aestivum) in South Africa: Stability analysis of yield performance. African J. Plant Soil. 17(3): 101-107. https://doi.org/10.1080/02571862.2000.10634878

Purchase, J.L. 1997. Parametric analysis to describe genotype $\mathrm{x}$ environment interaction and yield stability in winter wheat. Ph.D. Thesis, Department of Agronomy, Faculty of Agriculture, University of the Free State, Bloemfontein, South Africa. p. 48.

Sabaghnia, N., Dehghani, H. and Sabaghpour, S.H. 2008. Graphic analysis of genotype by environment interaction for lentil yield in Iran. Agron. J. 100(3): 760-764. https://doi.org/10.2134/agronj2006.0282

Shukla, G.K. 1972. Some statistical aspects of partitioning genotype-environmental components of variability. Heredity. 29(2): 237-245. https://doi.org/10.1038/hdy.1972.87
Simmonds, N.W. 1991. Selection for local adaptation in a plant breeding programme. Theor. Appl. Gene. 82(3): 363-367. https://doi.org/10.1007/BFo2190624

Sivetz, M. 1963. Coffee processing technology: Aromatization-properties-brewing decaffeination- plant design. Avi Publishing Company, Vol.2. Westport, Connecticut: p. 598.

Teressa, T., Semahegn, Z. and Bejiga, T. 2021 Multi Environments and GeneticEnvironmental Interaction (GxE) in Plant Breeding and its Challenges: a review article. Int. J. Res. Stud. Agril. Sci. 7(4): 11-18 https://doi.org/10.20431/2454-6224.0704002

Tollenaar, M. and Lee, E.A. 2002. Yield potential, yield stability and stress tolerance in maize. Field Crops Res. 75(2-3): 161-169. https://doi.org/10.1016/So378-4290(02)00024-2

Walyaro, D.J. 1983. Consideration in breeding for improved yield and quality in Arabica coffee $C$. arabica L. Wageningen, the Netherlands. pp. 88-92.

Wellman, F.L. 1961. Coffee: Botany, cultivation and utilization. Hill, London. 488p.

Wricke, G. 1964. The calculation of ecovalence in summer wheat and oat. $Z$ Pflanzenzuecht, 52: 127-138.

Yan, W. 2002 Singular value partitioning for biplot analysis of multi-environment trial data. Agron. J. 94(5): 990-996.

https://doi.org/10.2134/agronj2002.0990

Yan, W. and Hunt, L.A. 2001. Interpretation of genotype $\times$ environment interaction for winter wheat yield in Ontario. Crop Sci. 41(1): 19-25. https://doi.org/10.2135/cropsci2001.41119x

Yau S. K. 1995. Regression and AMMI analyses of genotype $\times$ environment interactions: An empirical comparison. Agron. J. 87(1): 121126.

https://doi.org/10.2134/agronj1995.0002196200 $8700010021 \mathrm{x}$

Zobel, R.W., Wright, M.J. and Gauch, H.G. 1988. Statistical analysis of a yield trial. Agron. J. 80(3): 388-393.

https://doi.org/10.2134/agronj1988.000219620 08000030002x 